\title{
INTEGRALS OF LOMMEL'S TYPE FOR CONFLUENT HYPERGEOMETRIC FUNCTIONS
}

\author{
by D. MARTIN
}

(Received 5th August, 1949)

In this note we derive some integrals involving confluent hypergeometric functions and analogous to Lommel's integrals for Bessel functions. Although the method of derivation is straightforward, the integrals do not seem to be mentioned in the literature.

1. The confluent hypergeometric functions $M_{k, m}(z), W_{k, m}(z)$ satisfy the differential equation (1)

$$
\frac{d^{2} w}{d z^{2}}+\left\{-\frac{1}{4}+\frac{k}{z}+\frac{\frac{1}{4}-m^{2}}{z^{2}}\right\} w=0
$$

When $2 m$ is not an integer, a fundamental system of solutions is formed by $\boldsymbol{M}_{k, m}(z)$ and $M_{k,-m}(z)$ or by $W_{k, m}(z)$ and $W_{-k, m}(-z)$, where

$$
M_{k, m}(z)=z^{\frac{1}{2}+m} e^{-\frac{1}{2} z}\left\{1+\frac{\frac{1}{2}+m-k}{(2 m+1) !} z+\ldots\right\},
$$

and

$$
W_{k, m}(z)=\frac{\Gamma(-2 m)}{\Gamma\left(\frac{1}{2}-m-k\right)} M_{k, m}(z)+\frac{\Gamma(2 m)}{\Gamma\left(\frac{1}{2}+m-k\right)} M_{k,-m}(z) .
$$

Let $u_{k, m}(z)$ and $u_{k^{\prime}, m^{\prime}}(z)$ be two solutions of (1.1), the particular type of function being left unspecified meantime. Then

$$
\begin{gathered}
\frac{d^{2}}{d z^{2}} u_{k, m}(\alpha z)+\alpha^{2}\left\{-\frac{1}{4}+\frac{k}{\alpha z}+\frac{\frac{1}{4}-m^{2}}{\alpha^{2} z^{2}}\right\} u_{k, m}(\alpha z)=0, \\
\frac{d^{2}}{d z^{2}} u_{k^{\prime}, m^{\prime}}(\beta z)+\beta^{2}\left\{-\frac{1}{4}+\frac{k^{\prime}}{\beta z}+\frac{\frac{1}{4}-m^{\prime 2}}{\beta^{2} z^{2}}\right\} u_{k^{\prime}, m^{\prime}}(\beta z)=0 .
\end{gathered}
$$

Multiplying (1.3) by $u_{k^{\prime}, m^{\prime}}(\beta z)$ and (1.4) by $u_{k, m}(\alpha z)$ and subtracting, we have

Hence

$$
\begin{aligned}
\frac{d}{d z}\left\{u_{k^{\prime}, m^{\prime}}(\beta z)\right. & \left.\frac{d}{d z} u_{k, m}(\alpha z)-u_{k, m}(\alpha z) \frac{d}{d z} u_{k^{\prime}, m^{\prime}}(\beta z)\right\} \\
& +\left\{-\frac{1}{4}\left(\alpha^{2}-\beta^{2}\right)+\frac{\alpha k-\beta k^{\prime}}{z}-\frac{m^{2}-m^{\prime 2}}{z^{2}}\right\} u_{k, m}(\alpha z) u_{k^{\prime} m^{\prime}}(\beta z)=0 .
\end{aligned}
$$

$$
\begin{array}{r}
\left.\int_{\lambda}^{\mu} \int^{1} \frac{1}{4}\left(\alpha^{2}-\beta^{2}\right)-\frac{\alpha k-\beta k^{\prime}}{z}+\frac{m^{2}-m^{\prime 2}}{z^{2}}\right\} u_{k, m}(\alpha z) u_{k^{\prime}, m^{\prime}}(\beta z) d z \\
=\left\{\alpha u_{k^{\prime}, m^{\prime}}(\beta z) u_{k_{,} m}^{\prime}(\alpha z)-\beta u_{k, m}(\alpha z) u_{k^{\prime}, m^{\prime}}(\beta z)\right\}_{\lambda}^{\mu},
\end{array}
$$

$\lambda, \mu$ being unspecified meantime.

2. We now apply (1.5) to the $M$-function. It follows from (1.2) that

$$
\begin{aligned}
\int_{0}^{z}\left\{\frac{1}{4}\left(\alpha^{2}-\beta^{2}\right)-\right. & \left.\frac{\alpha k-\beta k^{\prime}}{z}+\frac{m^{2}-m^{\prime 2}}{z^{2}}\right\} M_{k, m}(\alpha z) M_{k^{\prime}, m^{\prime}}(\beta z) d z \\
& =\alpha M_{k, m}(\beta z) M_{k, m}^{\prime}(\alpha z)-\beta M_{k, m}(\alpha z) M_{k^{\prime}, m^{\prime}}(\beta z),
\end{aligned}
$$


provided that, in general, $m+m^{\prime}>0$; if $m^{\prime}=m$, the condition is $m>-\frac{1}{2}$ and if, in addition, $k^{\prime}=k=0$, the condition is $m>-1$. In particular, if $k^{\prime}=k, m^{\prime}=m, \beta \neq \alpha$, the equation becomes

$$
\begin{aligned}
\int_{0}^{z}\left\{\frac{1}{4}(\alpha+\beta)\right. & \left.-\frac{k}{z}\right\} M_{k, m}(\alpha z) M_{k, m}(\beta z) d z \\
& =\frac{1}{\alpha-\beta}\left\{\alpha M_{k, m}(\beta z) M_{k, m}^{\prime}(\alpha z)-\beta M_{k, m}(\alpha z) M_{k, m}^{\prime}(\beta z)\right\}
\end{aligned}
$$

$m>-\frac{1}{2}$ (or $m>-1$, if $k=0$ ).

If $\beta=\alpha$ and the conditions $k^{\prime}=k, m^{\prime}=m$ are not both valid, (2.1) gives

$$
\begin{aligned}
\int_{0}^{z}\left\{\frac{\alpha\left(k^{\prime}-k\right)}{z}\right. & \left.+\frac{m^{2}-m^{\prime 2}}{z^{2}}\right\} M_{k, m}(\alpha z) M_{k^{\prime}, m^{\prime}}(\alpha z) d z \\
= & \alpha\left\{M_{k^{\prime}, m^{\prime}}(\alpha z) M_{k, m}^{\prime}(\alpha z)-M_{k, m}(\alpha z) M_{k^{\prime}, m^{\prime}}^{\prime}(\alpha z)\right\},
\end{aligned}
$$

with the same restrictions on $m, m^{\prime}$ as before.

We now consider the very special case $k^{\prime}=k, m^{\prime}=m, \beta=\alpha$. Putting $\beta=\alpha+\epsilon$ in (2.2) and expanding both sides of the equation in ascending powers of $\epsilon$, we have

$$
\begin{aligned}
\int_{0}^{z}\left\{\frac{1}{2} a+\frac{1}{4} \epsilon-\frac{k}{z}\right\} & \left\{M_{k, m}(\alpha z)+\epsilon z M_{k, m}^{\prime}(\alpha z)+\ldots\right\} M_{k, m}(\alpha z) d z \\
& =M_{k, m}(\alpha z) M_{k, m}^{\prime}(\alpha z)-\alpha z\left\{M_{k, m}^{\prime}(\alpha z)\right\}^{2}+\alpha z M_{k, m}(\alpha z) M_{k, m}^{\prime \prime}(\alpha z)+\text { terms in } \epsilon, \epsilon^{2} \text {, etc. }
\end{aligned}
$$

We now let $\epsilon$ tend to zero and obtain

$$
\int_{0}^{z}\left(\frac{\alpha}{2}-\frac{k}{z}\right)\left\{M_{k, m}(\alpha z)\right\}^{2} d z=M_{k, m}(\alpha z) M_{k, m}^{\prime}(\alpha z)-\alpha z\left\{M_{k, m}^{\prime}(\alpha z)\right\}^{2}+\alpha z M_{k, m}(\alpha z) M_{k, m}^{\prime \prime}(\alpha z), \ldots(2 .
$$

$m>-\frac{1}{2}$ (or $m>-1$, if $k=0$ ).

If, on the other hand, we put $k^{\prime}=k, m^{\prime}=m+\epsilon$ in (2.3), proceed as before and let $\epsilon$ tend to zero, we obtain

$$
\int_{0}^{z} \frac{\left\{M_{k, m}(\alpha z)\right\}^{2}}{z^{2}} d z=\frac{\alpha}{2 m}\left\{M_{k, m}(\alpha z) \frac{\partial}{\partial m} M_{k, m}^{\prime}(\alpha z)-M_{k, m}^{\prime}(\alpha z) \frac{\partial}{\partial m} M_{k, m}(\alpha z)\right\},
$$

$m>0$, or

$$
\int_{0}^{z} \frac{1}{z^{2}}\left\{M_{k, m}(z)\right\}^{2} d z=\frac{1}{2 m}\left\{M_{k, m}(z) \frac{\partial}{\partial m} M_{k, m}^{\prime}(z)-M_{k, m}^{\prime}(z) \frac{\partial}{\partial m} M_{k, m}(z)\right\}, \quad m>0 .
$$

Similarly, by putting $m^{\prime}=m, k^{\prime}=k+\epsilon$ in (2.3) we find that

$$
\int_{0}^{z} \frac{1}{z}\left\{M_{k, m}(z)\right\}^{2} d z=M_{k, m}^{\prime}(z) \frac{\partial}{\partial k} M_{k, m}(z)-M_{k, m}(z) \frac{\partial}{\partial k} M_{k, m}^{\prime}(z),
$$

$m>-\frac{1}{2}$ (or $m>-1$ if $k=0$ ).

(2.2), (2.3), (2.4) and (2.5) are analogous to Lommel's integrals for Bessel functions of the first kind. Indeed, using the relationship

$$
J_{n}(z)=\frac{M_{0, n}(2 i z)}{2^{2 n+\frac{1}{2}} i^{n+\frac{1}{2}} z^{\frac{1}{2}} \Gamma(n+1)},
$$

we can easily show that they lead to the results :

$$
\begin{aligned}
\left(\alpha^{2}-\beta^{2}\right) \int_{0}^{z} z J_{n}(\alpha z) J_{n}(\beta z) d z & =z\left\{\beta J_{n}(\alpha z) J_{n}^{\prime}(\beta z)-\alpha J_{n}(\beta z) J_{n}^{\prime}(\alpha z)\right\}, \quad n>-1, \\
\int_{0}^{1} J_{m}(\alpha z) J_{n}(\alpha z) \frac{d z}{z} & =\frac{\alpha}{m^{2}-n^{2}}\left\{J_{n}(\alpha) J_{m}{ }^{\prime}(\alpha)-J_{m}(\alpha) J_{n}^{\prime}(\alpha)\right\},
\end{aligned}
$$


$m+n>0, m \neq n$,

$$
\begin{aligned}
2 \alpha^{2} \int_{0}^{z} z\left\{J_{n}(\alpha z)\right\}^{2} d z & =\left(\alpha^{2} z^{2}-n^{2}\right)\left\{J_{n}(\alpha z)\right\}^{2}+\alpha^{2} z^{2}\left\{J_{n}^{\prime}(\alpha z)\right\}^{2}, \quad n>-1, \\
\int_{0}^{1}\left\{J_{n}(\alpha z)\right\}^{2} \frac{d z}{z} & =\frac{\alpha}{2 n}\left\{J_{n}(\alpha) \frac{\partial}{\partial n} J_{n}^{\prime}(\alpha)-J_{n}^{\prime}(\alpha) \frac{\partial}{\partial n} J_{n}(\alpha)\right\}, \quad n>0 .
\end{aligned}
$$

3. We now apply (1.5) to the $W$-function and take the limits of integration as $z$ and $\infty$. Since $W_{k, m}(z) \sim z^{k} e^{-\frac{1}{z} z}$ when $|z|$ is large and $|\operatorname{amp} z|<\pi$, we obtain

$$
\begin{aligned}
\int_{z}^{\infty}\left\{\frac{1}{4}(\alpha+\beta)-\frac{k}{z}\right\} & W_{k, m}(\alpha z) W_{k, m}(\beta z) d z \\
& =\frac{1}{\alpha-\beta}\left\{\beta W_{k, m}(\alpha z) W_{k, m}^{\prime}(\beta z)-\alpha W_{k, m}(\beta z) W_{k, m}^{\prime}(\alpha z)\right\},
\end{aligned}
$$

$\mathfrak{R}(\alpha+\beta)>0, \beta \neq \alpha$, and $\alpha, \beta, z$ being such that $\operatorname{amp}(\alpha z) \neq \pi$, amp $(\beta z) \neq \pi$.

The formula corresponding to $(2.4)$ is obviously

$\mathfrak{R}(\alpha)>0$.

$$
\begin{array}{r}
\int_{z}^{\infty}\left(\frac{1}{2} \alpha-\frac{k}{z}\right)\left\{W_{k, m}(\alpha z)\right\}^{2} d z=\alpha z\left\{W_{k, m}^{\prime}(\alpha z)\right\}^{2}-W_{k, m}(\alpha z) W_{k, m}^{\prime}(\alpha z) \\
-\alpha z W_{k, m}(\alpha z) W_{k, m}^{\prime \prime}(\alpha z),
\end{array}
$$

To avoid a possible singularity of the integrand, the path of integration in (3.1) and (3.2) should not pass through the origin.

The results corresponding to (2.3), (2.5) can be written down immediately and, with (3.1), (3.2), are the generalizations of the Lommel integrals for the modified Bessel function $K_{n}(z)$.

Analogous formulae for a certain class of $E$-functions can be obtained by means of the formula (2)

$$
E\left(\frac{1}{2}-k+m, \frac{1}{2}-k-m:: z\right)=\Gamma\left(\frac{1}{2}-k+m\right) \Gamma\left(\frac{1}{2}-k-m\right) z^{-k} e^{\frac{1}{z} z} W_{k, m}(z) .
$$

4. Equations (2.2) and (3.1) yield some information on the location of the zeros of the functions $M_{k, m}(z)$ and $W_{k, m}(z)$.

Suppose that $p+i q,(p, q$ real $)$ is a zero of $\boldsymbol{M}_{k, m}(z)$; then, since $k, m$ are regarded as being real, $p-i q$ must also be a zero. Hence, putting $\alpha=p+i q, \beta=p-i q$ in (2.2) and integrating along the real axis from 0 to 1 , we have

$$
\int_{0}^{1}\left(\frac{1}{2} p-\frac{k}{z}\right) M_{k, m}\{(p+i q) z\} M_{k, m}\{(p-i q) z\} d z=0
$$

$m>-\frac{1}{2}$ (or $m>-1$, if $k=0$ ).

Now the two $M$-functions in the integrand are conjugate complex numbers, so that their product is necessarily positive. Hence, if $k$ is positive, it follows that $p$ cannot be less than $2 k$, while if $k$ is negative, $p$ cannot exceed $2 k$. Also, if $k$ is zero, $p$ must be zero and the zeros of the function are purely imaginary (showing that the zeros of a Bessel function of the first kind are purely real). Thus, if

$m>-\frac{1}{2}, k>0$, there is no zero with real part less than $2 k$,

$m>-\frac{1}{2}, k<0$, there is no zero with real part greater than $2 k$,

$m>-1, k=0$, the zeros are purely imaginary.

Similarly, if $\alpha(=p+i q), \beta(=p-i q)$ are zeros of $W_{k, m}(z)$, (3.1) gives

$$
\int_{1}^{\infty}\left(-\frac{1}{2} p+\frac{k}{z}\right) W_{k, m}\{(p+i q) z\} W_{k, m}\{(p-i q) z\} d z=0, \quad p>0
$$


whence it follows that if $k \leqslant 0, W_{k, m}(z)$ has no zero with real part positive and that if $k>0$, $W_{k, m}(z)$ has no zero with real part greater than $2 k$. An immediate consequence of this is the well-known result that the modified Bessel function $K_{n}(z)$ has no zero with real part positive.

\section{REFERENCES}

(1) Whittaker \& Watson: Modern Analysis, Chapter XVI.

(2) MacRobert : Functions of a Complex Variable, 3rd edition, p. 351.

\section{UNIVERSTTY OF GLASGOW}

